\title{
Management of Complications During Video-Assisted Thoracic Surgery Lung Resection and Lymph Node Dissection
}

\author{
Yong Soo Choi, M.D. \\ Department of Thoracic and Cardiovascular Surgery, Samsung Medical Center, Sungkyunkwan University School of Medicine, Seoul, Korea
}

\section{ARTICLE INFO}

Received May 31, 2021

Revised July 27, 2021

Accepted July 27, 2021

Corresponding author

Yong Soo Choi

Tel 82-2-3410-1696

Fax 82-2-3410-6986

E-mail choi.smcts@skku.edu

ORCID

https://orcid.org/0000-0002-2552-0067

\begin{abstract}
Intraoperative events can occur during video-assisted thoracoscopic surgery (VATS) lobectomy due to unfavorable surgical anatomy, such as dense adhesions or calcifications around the pulmonary arteries. Troubleshooting intraoperative complications is essential for performing safe and successful VATS pulmonary resection and lymph node dissection. If continuous bleeding occurs or VATS does not proceed despite all measures, conversion to open thoracotomy should not be delayed.
\end{abstract}

Keywords: Video-assisted thoracoscopic surgery, Intraoperative events, Complication

\section{Introduction}

Video-assisted thoracoscopic surgery (VATS) pulmonary resection and lymph node dissection is a widely accepted technique for early-stage non-small cell lung cancer [1,2]. A learning curve is required to perform VATS lobectomy for lung cancer. VATS lobectomy is known to be associated with a significantly higher rate of intraoperative complications than open thoracotomy lobectomy [3]. Intraoperative events can occur during VATS lobectomy when performed by either an experienced surgeon or a novice surgeon due to unfavorable surgical anatomy, such as dense adhesions or calcifications around the pulmonary arteries. Troubleshooting intraoperative complications is essential for performing safe and successful VATS pulmonary resection and lymph node dissection. This article reviews the causes, related factors, and troubleshooting and prevention strategies for intraoperative events during VATS.

\section{Bleeding}

Significant unexpected bleeding mainly occurs due to injuries of the pulmonary arteries, pulmonary veins, and bronchial arteries. Inappropriate and unclear exposure and careless dissection, ligation, or division of vessel structures were found to be the most common causes of vascular in- juries [4]. Stapler or device malfunction leading to bleeding is relatively rare, and most cases are likely related to the surgeon's error [5].

The most common bleeding sites during VATS are the pulmonary arteries. It is recognized that patients receiving neoadjuvant chemotherapy and/or radiation therapy and those with larger tumors are at greater risk for an arterial injury [6,7]. The presence of calcified lymph nodes between the pulmonary artery and bronchus causes bleeding issues, especially in older patients with previous inflammatory disease. Pulmonary vein injuries are much less common than pulmonary artery injuries. Careless dissection of the inferior pulmonary ligament can cause injuries to the inferior pulmonary vein. Bleeding from the bronchial arteries is common during VATS, but can be easily controlled by compression, electrocautery, ultrasonic energy devices, or hemoclips. However, postoperative bleeding from the bronchial arteries, especially along the bronchial stump or subcarinal area, requires reoperation for bleeding control.

Appropriate exposure and detection of anatomical structures with attention to careful and meticulous vascular dissection, ligation, and division are the most critical elements for avoiding serious injuries [8]. Inadequate exposure of the hilar structures is rather common when vision is provided from the lower port below the sixth intercostal space. Three-dimensional-view VATS provides better visu- 
alization than 2-dimensional VATS $[9,10]$, enabling more comfortable identification and dissection of vascular structures.

A basic safety strategy is to keep a sponge or a clamp on the back table as preparation for sudden unexpected bleeding during any minimally invasive surgery. The instant goal is the immediate control of hemorrhage and restoring the view of the operative field. Direct compression using a sponge stick or the surrounding lung is an effective way to stop or decrease bleeding from injured vessels. However, in cases of massive bleeding, it may not be easy to identify the bleeding focus. After sufficient compression for at least a few minutes, while maintaining a stable blood pressure and heart rate, the decision should be made regarding how to control bleeding and continue with the next step of the procedure. Conversion to open thoracotomy should also be considered if the vital signs are unstable due to continuous bleeding. Compression itself, electrocautery, hemostatic clips, and other hemostatic agents may also be used. A wet fibrin sealant patch is placed over the bleeding focus and compressed gently for at least a few minutes. This maneuver is usually enough to stop bleeding. If bleeding continues, vascular clamping of the proximal portion of the bleeding focus with an endoscopic vascular clamp is the next step, but it is difficult for novice surgeons to control the bleeding with a vascular clamp. After successfully clamping the proximal part of the injured vessel, primary repair with a suture could be done. Increasing the number of VATS ports may be helpful to obtain better exposure of the injured vessels and control the bleeding.

A novel method for managing vascular injuries, called the suction-compression angiorrhaphy technique, involves first controlling bleeding by side-compression of the injured site with endoscopic suction. Then, angiorrhaphy is performed with a running suture, with the specific procedure depending on the size and location of the injuries; options include direct suturing upon suction compression, suturing after substituting suction compression with clamping of the injured site, or suturing after attaining proximal cross-clamping of the main pulmonary artery [11].

If bleeding continues despite all measures, conversion to open thoracotomy should not be delayed. Compression with a sponge stick over the bleeding focus should be maintained via the lower port when making the thoracotomy extension.

\section{Bronchus injury, fused interlobar fissure, and air leak}

Bronchial injuries occurred in $6(0.94 \%)$ of 633 patients who underwent VATS anatomic lung resection, requiring 3 unplanned pneumonectomies and 1 unplanned bilobectomy, and causing 1 tracheoesophageal fistula and 1 membranous airway injury to the bronchus intermedius [3]. Direct injury to the bronchus by electrocautery or an ultrasonic device is clearly identified during the air leak test after the completion of lung resection, but it is not possible to check intraoperatively for a delayed bronchial injury caused by a thermal mechanism. A minimal injury to the membranous portion of the bronchus or an inadequately stapled stump of the bronchus can become aggravated to form a bronchopleural fistula in patients with diabetes mellitus or chronic steroid usage. Reinforcement of the bronchial stump with pericardium or a fibrin sealant patch over the bronchial stump and neighboring bronchus could decrease the risk of bronchial fistula after the operation.

When lobectomy is performed in a patient with a fused interlobar fissure, there is a higher risk of air leakage [12]. There is no consensus regarding the choice between a fissure-last technique [13] and a fissure-first technique [14] during VATS lobectomy. Careful identification of the pulmonary vein pathway is required in cases of right upper lobectomy. Some portions of the middle lobe and lower lobe drained into the superior pulmonary vein via the upper pulmonary vein, so careless transection of those tributaries may result in congestion of the middle lobe and lower lobe after right upper lobectomy. Therefore, a fissure-first technique is recommended in right upper lobectomy. A totally fused, thick interlobar fissure is a reason for conversion to open thoracotomy.

Prolonged air leak is a major reason for long hospital stays and remains a common complication after VATS lobectomy. Several risk factors for prolonged air leak have been identified, including old age, a body mass index under $24.0 \mathrm{~kg} / \mathrm{m}^{2}$, the surgeon's experience, the surgical site (upper lobectomy and bilobectomy), decreased pulmonary function, fused fissure, and pleural adhesions $[15,16]$. Meticulous care should be taken during the division of a fused fissure and pleural adhesiolysis in patients with these factors. 


\section{Miscellaneous tips to manage and prevent intraoperative events}

(1) Postoperative delayed gastric emptying could be minimized by taking care not to damage the vagus nerve, especially when dissecting the subcarinal nodes and aortopulmonary nodes. Meticulous care should be taken when performing lobectomy and node dissection in patients who have previously undergone contralateral lobectomy and node dissection.

(2) Avoid damaging the recurrent laryngeal nerve when dissecting the right upper paratracheal nodes and left lower paratracheal nodes and aortopulmonary nodes.

(3) Injuries of the diaphragm or esophagus are indolent and subclinical during the operation or the immediate postoperative days.

(4) The anvil tip of endoscopic staplers is slightly traumatic, so care should be taken when manipulating staplers.

(5) Conversion to open thoracotomy should not be considered a source of shame to the surgeon. Even experts need to convert to thoracotomy when facing a difficult situation during VATS lobectomy. Please do not forget that VATS is not a goal of surgery itself, but instead one of multiple approach techniques.

\section{Conflict of interest}

No potential conflict of interest relevant to this article was reported.

\section{ORCID}

Yong Soo Choi: https://orcid.org/0000-0002-2552-0067

\section{References}

1. Ettinger DS, Akerley W, Borghaei H, et al. Non-small cell lung cancer, version 2.2013. J Natl Compr Canc Netw 2013;11:645-53.

2. Gopaldas RR, Bakaeen FG, Dao TK, Walsh GL, Swisher SG, Chu D. Video-assisted thoracoscopic versus open thoracotomy lobectomy in a cohort of 13,619 patients. Ann Thorac Surg 2010;89:1563-70.

3. Flores RM, Ihekweazu U, Dycoco J, et al. Video-assisted thoracoscopic surgery (VATS) lobectomy: catastrophic intraoperative com- plications. J Thorac Cardiovasc Surg 2011;142:1412-7.

4. Louie BE. Catastrophes and complicated intraoperative events during robotic lung resection. J Vis Surg 2017;3:52.

5. Brown SL, Woo EK. Surgical stapler-associated fatalities and adverse events reported to the Food and Drug Administration. J Am Coll Surg 2004;199:374-81.

6. Decaluwe H, Petersen RH, Hansen H, et al. Major intraoperative complications during video-assisted thoracoscopic anatomical lung resections: an intention-to-treat analysis. Eur J Cardiothorac Surg 2015;48:588-99.

7. Augustin F, Maier HT, Weissenbacher A, et al. Causes, predictors and consequences of conversion from VATS to open lung lobectomy. Surg Endosc 2016;30:2415-21.

8. Safdie FM, Sanchez MV, Sarkaria IS. Prevention and management of intraoperative crisis in VATS and open chest surgery: how to avoid emergency conversion. J Vis Surg 2017;3:87.

9. Dong S, Yang XN, Zhong WZ, et al. Comparison of three-dimensional and two-dimensional visualization in video-assisted thoracoscopic lobectomy. Thorac Cancer 2016;7:530-4.

10. Kim TH, Hong TH, Choi YS. Comparison of surgical outcomes between $3 D$ and $2 D$ VATS lobectomy for clinical stage I lung cancer. Precis Future Med 2020;4:69-74.

11. Mei J, Pu Q, Liao H, Ma L, Zhu Y, Liu L. A novel method for troubleshooting vascular injury during anatomic thoracoscopic pulmonary resection without conversion to thoracotomy. Surg Endosc 2013;27:530-7.

12. Igai H, Kamiyoshihara M, Yoshikawa R, et al. The efficacy of thoracoscopic fissureless lobectomy in patients with dense fissures. J Thorac Dis 2016;8:3691-6.

13. Balsara KR, Balderson SS, D'Amico TA. Surgical techniques to avoid parenchymal injury during lung resection (fissureless lobectomy). Thorac Surg Clin 2010;20:365-9.

14. Decaluwe H, Sokolow Y, Deryck F, et al. Thoracoscopic tunnel technique for anatomical lung resections: a 'fissure first, hilum last' approach with staplers in the fissureless patient. Interact Cardiovasc Thorac Surg 2015;21:2-7.

15. Rivera C, Bernard A, Falcoz PE, et al. Characterization and prediction of prolonged air leak after pulmonary resection: a nationwide study setting up the index of prolonged air leak. Ann Thorac Surg 2011;92:1062-8.

16. Zhao K, Mei J, Xia C, et al. Prolonged air leak after video-assisted thoracic surgery lung cancer resection: risk factors and its effect on postoperative clinical recovery. J Thorac Dis 2017;9:1219-25. 